\title{
Les valeurs au cœur du langage
}

Values at the Heart of Language

Jean-Claude Guerrini

\section{OpenEdition}

Journals

Édition électronique

URL : http://journals.openedition.org/recherchestravaux/1617

DOI : 10.4000/recherchestravaux.1617

ISSN : 1969-6434

\section{Éditeur}

UGA Éditions/Université Grenoble Alpes

\section{Édition imprimée}

ISBN : 978-2-37747-098-3

ISSN : 0151-1874

\section{Référence électronique}

Jean-Claude Guerrini, "Les valeurs au cœur du langage », Recherches \& Travaux [En ligne], 94 | 2019, mis en ligne le 20 juin 2019, consulté le 08 septembre 2020. URL : http://journals.openedition.org/ recherchestravaux/1617; DOI : https://doi.org/10.4000/recherchestravaux.1617

Ce document a été généré automatiquement le 8 septembre 2020

(C) Recherches \& Travaux 


\title{
Les valeurs au cœur du langage
}

\author{
Values at the Heart of Language
}

Jean-Claude Guerrini

\section{Introduction}

1 Il est bon, assurément, de se donner pour but de transmettre et de questionner les valeurs. Encore faut-il savoir comment on les identifie, comment on les situe les unes par rapport aux autres, bref, comment on peut avoir une idée d'ensemble de la dimension axiologique des discours. Les philosophes, les sociologues, les anthropologues offrent leurs services, à partir des exigences et de la méthodologie de leur discipline. Le propos de cet article est de montrer que la question des valeurs peut être abordée à partir de ce qui constitue le cœur de la discipline du français: l'enseignement de la langue et l'étude des textes.

2 Lorsque Chaïm Perelman, au lendemain de la Seconde Guerre mondiale, soucieux de proposer une approche rationnelle de la question des valeurs, mena une enquête sur leurs manifestations discursives pour répondre à ses interrogations de philosophe du droit et d'ancien résistant ${ }^{1}$, il ne vit d'autre voie que de recourir aux services de la rhétorique, une discipline tombée en désuétude à un point qu'il nous est aujourd'hui difficile d'imaginer'2. Dans le Traité de l'argumentation, la nouvelle rhétorique, co-écrit avec Lucie Olbrechts-Tyteca ${ }^{3}$, il se tourna vers les ressources de l'antiquité, tout en étant bien conscient du caractère proprement moderne de la question des valeurs. Mais, disparu en 1984, il n'a pu disposer des ressources de la pragmatique, de la linguistique énonciative et de la sémiotique.

3 À la suite des travaux d'A. J. Greimas, de Catherine Kerbrat-Orecchioni et de Vincent Jouve, il est aujourd'hui possible de proposer une approche intégrative de la dimension axiologique des discours, interne à la discipline littéraire, même si les apports d'autres disciplines ne sauraient en aucun cas être négligés.

Qu'on ne se méprenne pas. Comme le garantit notre ancrage dans la tradition rhétorique, il ne s'agit ici nullement de reconduire une approche formaliste qui durant 
de nombreuses années a conduit à détourner les lecteurs, les élèves et les étudiants en lettres de l'attention au sens et à les priver d'une rencontre sensible et compréhensive avec les œuvres. C'est au contraire, pour faciliter l'accès à la substance des textes, à leur complexité et à leurs enjeux, qu'on présentera ici de manière forcément trop condensée quelques éléments d'une topique résultant de l'observation de la langue et du fonctionnement des textes, littéraires ou non ${ }^{4}$.

\section{Quelques mises au point historiques}

Il importe tout d'abord de situer historiquement les notions de "jugement de valeur » et de « valeurs ».

Le "jugement de valeur » est une notion qui n'apparaît qu'à la fin du XIX siècle en opposition au jugement de fait ou au jugement de réalité. Auparavant, les jugements normatifs n'étaient guère distingués de la vérité (notamment quand ils étaient pris en charge) ou se trouvaient rangés dans la catégorie peu recommandable des opinions. Longtemps la notion de jugement de valeur n'a été répertoriée que dans les dictionnaires philosophiques. Ce n'est que dans les années $1960 \mathrm{du} \mathrm{XX}^{\mathrm{e}}$ siècle qu'elle apparaît dans le dictionnaire Le Robert, en même temps que la lexie valeurs (en tant que pluriel abstrait) fait l'objet d'un traitement séparé dans l'entrée "valeur » pour désigner : «ce qui est vrai, beau, bien, selon un jugement personnel, plus ou moins en accord avec celui de l'époque » (Dictionnaire Le Robert, 1964). La première attestation de valeurs dans ce sens est attribuée à Hippolyte Taine dans un cours sur L'Idéal dans l'art de 1867.

7 Il est donc indispensable de garder à l'esprit le caractère anachronique de l'usage de cette lexie valeurs quand on l'applique à des auteurs antérieurs (qu'il s'agisse de Platon, de Corneille ou de Victor Hugo). Platon, usant du neutre d'adjectifs substantivés pour nommer le bien, le beau, le vrai, parlait à leur propos des « choses les plus hautes ou les plus grandes » $(\text { megista })^{5}$. Sous le nom de topoi ou de lieux de l'argumentation (loci), les traités de rhétorique grecs et romains désignaient les critères du jugement susceptibles d'intervenir de manière privilégiée dans telle ou telle circonstance (l'utile devant une assemblée, le juste au tribunal, le beau dans les discours d'apparat). Le vocabulaire des Idées platoniciennes, des vertus (païennes, puis chrétiennes), l'usage de ces "grand mots » (souvent à majuscules au xIX ${ }^{e}$ siècle) que nous appellerons les maitres-mots ont précédé l'apparition du terme valeurs dont on voit qu'il fournit un instrument commode pour caractériser des convictions ou des familles de jugements partagés par un groupe ou une communauté (valeurs classiques, valeurs romantiques, valeurs de la Résistance, valeurs européennes, valeurs de la droite, valeurs de la gauche, valeurs du sport, etc.). C'est pourquoi, il est légitime d'user de cette lexie, en pratiquant un " anachronisme contrôlé ${ }^{\text {", }}$, pour caractériser les instruments normatifs des sociétés dans lesquelles elle n'était pas employée. On doit remarquer, par ailleurs, que ce terme est devenu d'un usage courant dans les relations interculturelles, pour désigner des motivations autres que les seuls intérêts.

8 L'usage du mot axiologie $e^{7}$, que la sémiotique et la linguistique de l'énonciation ont diffusé en même temps que ses dérivés (axiologique, axiologiser, axiologisation) contribue à donner plus de souplesse aux analyses attentives au jeu des valeurs dans les textes. L'adjectif axiologique est particulièrement précieux dans la mesure où il peut être considéré comme l'adjectif relationnel manquant du mot valeur (de axios : qui est de 
poids, qui vaut ; qui mérite, digne de ; qui en vaut la peine ; de grande valeur selon le dictionnaire Grec-Français Bailly).

9 L'essor du mot valeur qui est lié évidemment à son usage en économie (ce qui suffit pour certains à le disqualifier) a été suivi de celui de toute une série de dérivés (valorisation, dévalorisation, survalorisation, valorisant, dévalorisant) qui, apparus dans la sphère économique, ont progressivement été employés dans le domaine de la psychologie ou des sciences sociales, pour devenir bientôt des termes servant à caractériser d'une manière générale des variations de valeur et de statut (on parle par exemple en lexicologie de termes "valorisants » ou " dévalorisants »). La coloration de certains de ces termes dépend de l'air du temps : c'est ainsi que la «valorisation » a tendance à désigner actuellement en priorité, dans les organisations et les entreprises, une forme de promotion institutionnelle ou économique des produits de la recherche. Mais la prise en compte du contexte et du genre de texte, permet de l'employer dans un sens plus large, comme le font les auteurs du Traité de l'argumentation.

\section{Les principaux axes du jugement de valeur}

10 La mise au jour de la dimension axiologique des discours est d'autant plus délicate qu'elle doit prendre en compte les données explicites fournies par les termes ouvertement axiologiques (tels les adjectifs bien, bon, beau, etc., ou les mots-valeurs tels que justice, égalité, altruisme, etc.), mais aussi des données implicites qui peuvent être identifiées par inférence à partir des tensions intervenant dans la trame des textes du fait des choix lexicaux, des trajets argumentatifs ou narratifs, des parcours émotionnels proposés au lecteur.

11 Ce jeu de l'explicite et de l'implicite (qui peut laisser sa place à un « effacement » plus ou moins abouti du jugement de valeur) produit un « relief axiologique » qui offre des prises plus ou moins propices à la contestation et à la réfutation.

12 Commençons par ce qui constitue une sorte de cadre sémantique général à l'intérieur duquel tendent à se loger l'ensemble de nos jugements de valeur. En tenant compte des recherches portant sur la subjectivité dans le langage ${ }^{8}$ et sur le système des modalités du français ${ }^{9}$, il nous paraît en effet envisageable de parvenir à une clarification concernant l'espace sémantique qui conditionne et rend possibles l'immense majorité de nos évaluations.

13 Catherine Kerbrat-Orecchioni, pour mener la première étude systématique sur l'expression de la subjectivité dans le langage, situait les axiologiques (adjectifs, noms, verbes, adverbes) au sein d'un continuum entre termes "objectifs» et termes "subjectifs", les axiologiques constituant la catégorie la plus "subjective». Elle distinguait nettement modalité axiologique et modalité épistémique, mais rappelait que tout énoncé aléthique (référant à la "vérité objective ») devait, compte tenu de son inévitable «situation » dans une interaction présupposant locuteur et destinataire, se reformuler en termes épistémiques.

14 Laurent Gosselin, quant à lui, reprend à son compte l'idée de Charles Bally selon laquelle il importe de "dissocier le locuteur (ou "sujet parlant") du "sujet modal", conçu comme l'instance responsable du jugement ${ }^{10} »$. Il définit alors les modalités comme « ce qui doit être ajouté aux représentations pour qu'il y ait constitution d'un jugement ${ }^{11} »$. Il en fournit une liste close (aléthique, épistémique, appréciative, 
axiologique, boulique, déontique) accompagnée d'un inventaire détaillé de leurs multiples moyens d'expression: lexèmes, coverbes modaux, adverbes modaux, périphrases verbales, mais aussi inférences dérivant de stéréotypes ou liées à des situations de discours. Il est remarquable pour notre propos que Gosselin trouve utile de rattacher ces strates profondes de l'activité discursive aux genres oratoires et à leurs buts principaux, ces topoï en considération desquels se développent les divers genres du discours: "le délibératif qui vise principalement à conseiller/déconseiller et dont la modalité essentielle est celle de l'utile/nuisible; le judiciaire qui consiste à accuser/ défendre sur la base de la modalité du juste et de l'injuste; et enfin l'épidictique caractérisé par la force illocutoire de la louange et du blâme associé à la modalité du beau/laid (au sens moral) ${ }^{12} »$.

Il existe un lien indéniable entre les modalités, telles que Gosselin les caractérise et les axes de la (dé)valorisation que nous souhaitons identifier :

Très schématiquement, les modalités épistémiques (certain, probable, contestable...) suscitent les croyances (et les doutes), c'est-à-dire qu'elles sont censées entraîner la conviction; alors que les modalités appréciatives et axiologiques provoquent les désirs (et les aversions), qui, joints à la conviction, constituent la persuasion, en tant que visée perlocutoire globale du discours ${ }^{13}$.

Mais les découpages auxquels Gosselin est conduit ne nous semblent pas toujours appropriés. Reprenant une certaine tradition philosophique, il réduit la modalité axiologique à la catégorie bien/mal et au discours moral/éthique, alors que depuis les travaux de Greimas, de Kerbrat-Orecchioni et de Galatanu, les axiologiques désignent la zone plus large des termes "péjoratifs (dévalorisants)/mélioratifs (laudatifs, valorisants) » et, d'une façon générale, l'espace sémantique qui relève de l'archi-axe B/ M (bon/mauvais) indépendamment des spécifications qui lui sont apportées. D'autre part, Gosselin présente la modalité appréciative de telle manière qu'elle constitue une catégorie très étendue qui rassemble des jugements hétérogènes (beau, efficace, agréable) relevant du désirable et reliée, par le moyen de métarègles, à l'axiologique.

Comme, par ailleurs, malgré les proximités qu'elles entretiennent avec les critères des jugements de valeur, les modalités aléthique (établissant une vérité «objective » du type vrai/faux), déontique (du type «on doit») et volitive (du type "je veux»), ne peuvent entrer directement dans le champ de l'axiologique, il nous paraît indispensable de ménager, en lien avec le plan des modalités, un plan des valeurs pourvu d'une certaine autonomie.

cette perspective les propositions de Patrick Charaudeau dans sa Grammaire du sens et de l'expression ${ }^{14}$ méritent d'être examinées avec soin. Pour la première fois dans ce type de manuels, elles présentent pour l'étude du mode d'organisation argumentatif, au titre de "procédés sémantiques", les valeurs et un ensemble de cinq "domaines d'évaluation » qui proposent un cadre utile : le domaine de la Vérité (vrai/faux), le domaine de l'Esthétique (beau/laid), le domaine de l'Éthique (bien/mal), le domaine de l'Hédonique (agréable/désagréable) et le domaine du Pragmatique (utile/inutile).

19 Cependant, la présence des majuscules allégorisantes témoigne de la persistance d'une conception platonisante des valeurs. Par ailleurs, le «domaine de la vérité » se voit caractérisé de manière peu claire :

Le domaine de la Vérité, qui définit de manière absolue, et en termes de vrai et de faux, d'une part l'existence des êtres dans leur originalité, leur authenticité et leur unicité, d'autre part ce qui relève du savoir comme principe unique d'explication des phénomènes du monde ${ }^{15}$. 

vérité envisagée dans sa dimension épistémique selon une échelle du vraisemblable au vrai, du plausible au certain, du douteux à l'avéré, on entretient l'idée paradoxale d'une vérité qui n'aurait rien à voir avec les valeurs, car relevant uniquement de l'opposition vrai/faux. Il nous semble au contraire indispensable de distinguer l'aléthique et l'épistémique afin de ne pas confondre la vérité comme résultat d'un jugement de fait ou de réalité et la vérité comme réponse à une exigence interprétative de cohérence et de signification. La quête de la certitude relève d'une aspiration, non d'un constat. C'est pourquoi l'on peut parler de la vérité comme d'un fait, en termes aléthiques et comme d'une valeur, en termes épistémiques.

En même temps que nous choisissons le qualificatif d'épistémique pour ce type de jugement axiologique, nous substituons au mot de « domaine » celui d'« axe », qui évite de suggérer l'idée d'ensembles étanches ${ }^{16}$. Nous considérons ces 5 axes, auxquels nous ôtons leur caractère inutilement emphatique, comme spécifiant le jugement de valeur, en réservant le cas où, prenant une autre forme (v/v'), l'opposition bon/mauvais investirait une autre opposition jugée centrale (par exemple l'opposition vie/mort ou pur/impur). La constance de ces 5 axes, d'un usage somme toute ordinaire, leur donne néanmoins le statut de conditions a priori du jugement, constatées empiriquement dans les pratiques langagières de la culture occidentale depuis plus de deux millénaires. De même que toute énonciation suppose un locuteur, un destinataire et un cadre spatiotemporel, de même tout jugement de valeur se situe dans un cadre sémanticopragmatique contraint par ces axes de (dé)valorisation ${ }^{17}$ dont la présence explicite ou implicite vient étayer la prétention à la légitimité, notamment, et cela peut paraître paradoxal, quand la revendication de singularité et d'indépendance à l'égard des normes communes cherche à s'affirmer.

22 Le cas de Sade est à cet égard éclairant. Loin de se situer dans un cadre de justification étranger à la morale, il s'y inscrit continûment, ne faisant qu'inverser les polarités au nom d'une supériorité du plaisir sur la religion (et la morale conformiste qui y est associée), comme du mal sur le bien. Comme le note Gosselin ${ }^{18}$, il y a chez les personnages sadiens, un plaisir particulier à faire le mal. Mais cela ne signifie pas que l'on ne juge plus en termes de bien/mal, tout au contraire : le plaisir sinon disparaîtrait.

De la même façon, le fonctionnement de Modest Proposal de J. Swift ${ }^{19}$, qui accumule les indices axiologiques de l'utilité en faisant l'impasse absolue sur les considérations morales ou éthiques, repose justement sur l'attente inévitable de celles-ci chez le destinataire. Le locuteur "utilitariste » mis en scène par l'auteur, indifférent au sort des nourrissons qu'il propose à la consommation de ses concitoyens pour régler le problème irlandais, ne peut que scandaliser: l'axe moral-éthique, qui ne relève pas seulement de l'imposition d'une norme institutionnelle, exercera nécessairement sa vigilance devant la transgression et le projet de massacre qui se dessine.

24 L'agacement et l'ironie à l'égard des "valeurs ", surtout des plus générales, quand on est amené à les expliciter, s'expliquent par la prétention à la liberté de jugement qui anime les individus des sociétés démocratiques. Mais, de même que tout acte libre s'inscrit dans le temps et dans l'espace, tout jugement se détermine, à tout le moins, en fonction des axes de la (dé)valorisation qui lui permettent de se formuler. Cela signifiet-il que tout jugement se référera à ces axes ? Évidemment non. Ce n'est que dans une situation de crise ou de choix crucial que l'inscription de tel prédicat ou de telle 
opposition sémantique dans un contexte donné cherchera à se doter d'un garant ultime en invoquant l'un d'entre eux.

Nous proposons par conséquent, comme résultat de ces remarques, une représentation synthétique de la dimension axiologique destinée à faire apparaître sa cohérence et sa relative autonomie par rapport au système d'ensemble des modalités.

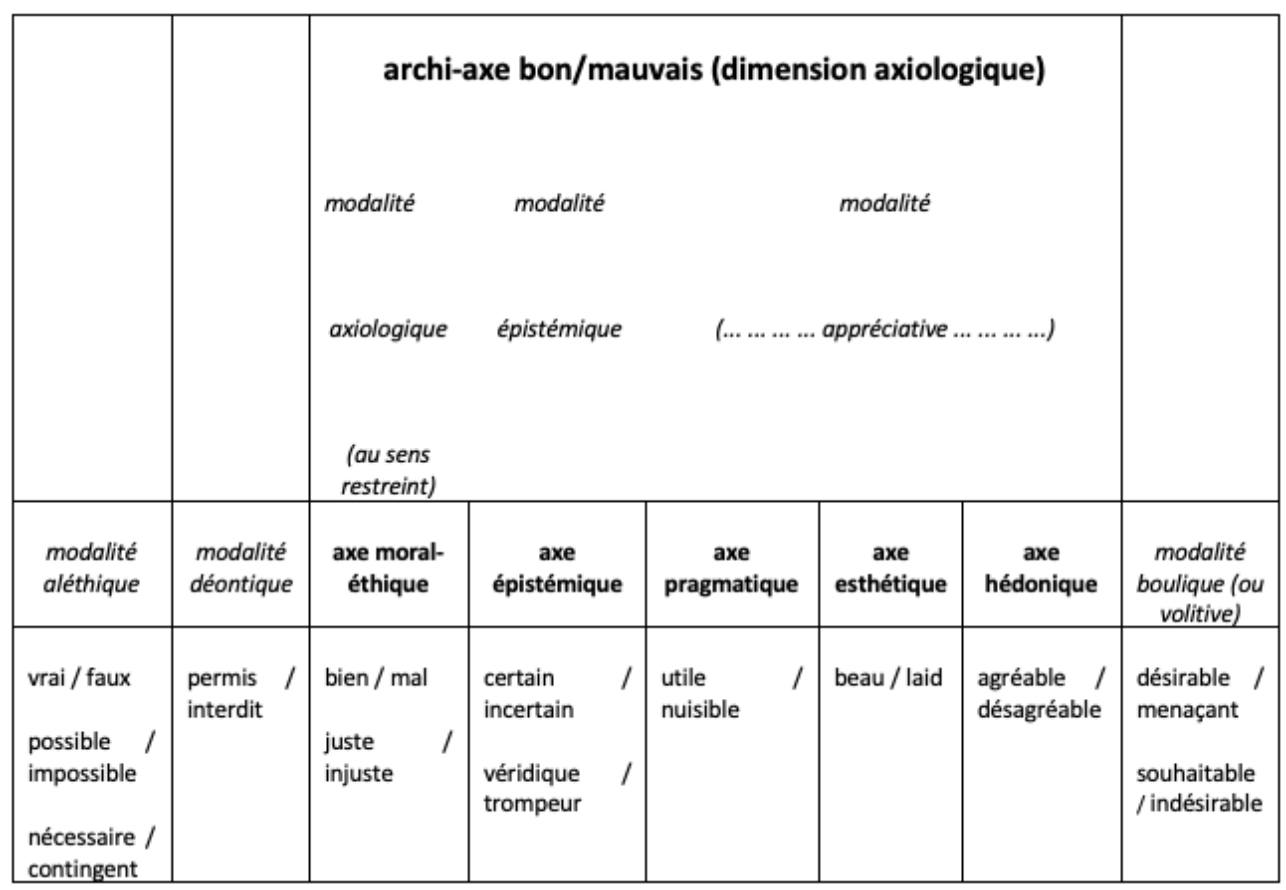

Fig. 1 Principaux axes de la (dé)valorisation et modalités

Les axes sont mentionnés en gras, les modalités distinguées par L. Gosselin apparaissant en italique ${ }^{20}$.

\section{Les maîtres-mots ou mots-valeurs}

Les maîtres-mots (" honneur », « liberté », " solidarité », « respect », « esprit sportif », etc.) ne sont pas que des mots-clés. Ils constituent des garants au nom desquels on s'estime habilité à porter des jugements d'approbation ou de blâme (des jugements de valeur), à justifier une préférence, à prendre position, à agir. Ils servent de médiation entre les participants d'une interaction, qu'ils contribuent à motiver des droits ou des sanctions, à constituer des consensus ou à définir des postures de refus ou de résistance.

Certains noms ont pour fonction essentielle d'indiquer ce qui est bon, souhaitable dans un domaine particulier de la conduite. Bien sûr, le même objectif peut être atteint par d'autres voies et l'expression des valeurs ne se confond pas avec l'emploi de ces motsdrapeaux. Mais leur usage est suffisamment reconnu pour que la notion de valeur leur soit le plus souvent associée. "Fidélité", "tolérance", "tradition», "savoir", " générosité ", " élégance ", " travail », "sens du devoir ", " respect de la nature ", " authenticité ", «solidarité », etc. formulent chacun une certaine idée du préférable. Ils présupposent, sans qu'un lexème vienne toujours leur correspondre, un contraire, les anti-valeurs. 

l'école, les médias, etc.), dont l'ambition, plus ou moins revendiquée, est de fournir des normes de jugement, des schémas d'attitudes, de comportements, de pratiques. Ils condensent des composantes de formes de vie, des normes de conduite dont l'inventaire peut être des plus utiles. D'autre part, ils manifestent un rapport aux mots et au langage (confiance/défiance; révérence/dérision) qu'il paraît utile d'analyser si l'on désire approfondir la connaissance des liens entre langue et culture. Consciemment ou non, leur maniement participe de la construction de l'identité des individus ou des groupes, mais affirmer le caractère absolu des valeurs, dire qu'elles ne sont pas négociables, relève souvent de la posture, l'expérience contraignant habituellement à des compromis sauf dans des cas limites de radicalisation.

On peut distinguer cinq modes d'intervention des valeurs dans un discours en fonction $\mathrm{du}$ rapport plus ou moins étroit qu'elles entretiennent, dans l'énonciation, avec le locuteur ou le destinataire : la convocation, l'invocation, la préconisation, l'évocation et la mention.

\section{Fonctions pragmatiques des mots-valeurs}

\section{Invocation (ou démystification)}

L'usage de la locution prépositive «Au nom de...» est révélateur de ce type de fonctionnement. Destinée tout d'abord à indiquer une relation d'allégeance ou de délégation (Au nom de Dieu, du Roi, du peuple français, de la loi, etc.), elle en est venue à exprimer une référence à des "valeurs" censées inspirer l'action. Formulation typique de l'argument d'autorité, elle renvoie, en surplomb, à une instance qui légitime une conduite ou une prise de position :

- «Les soussignés protestent, au nom de la liberté de pensée et de la liberté de parole, contre la suspension du cours du citoyen Michelet, et chargent les représentants du peuple, auxquels ils transmettront cette protestation, de la défendre à la tribune. » (Jules Vallès, Le Bachelier, 1881);

- «La seule chose qui compte, c'est de savoir, si l'invention qui se fait, se fait au nom de la liberté. » (Jean-Paul Sartre, L'Existentialisme est un humanisme, 1946).

31 Cette théatralisation de la médiation invite au respect et peut s'accompagner d'une forme d'intimidation, le contenu pris en charge servant de rempart contre toute mise en cause. Mais dans la mesure où, précisément, elle met à nu les motifs invoqués, cette formule favorise la démystification. Le soupçon peut en effet porter sur l'authenticité de la référence ou la qualité de sa mise en pratique.

Il est possible de faire un usage herméneutique de cette locution. Moyennant quelques précautions, elle peut servir d'« extracteur de valeur ", en posant la question « au nom de quoi ? » à celui dont on exige une justification. Mais, la locution en étant venue à véhiculer tout type d'argument, il faut accompagner la question de la vérification suivante : « au nom de quelle valeur ${ }^{21}$ ? », et s'assurer que le mot-valeur (V) peut :

- entrer dans un syntagme du type la valeur $\mathrm{V}$ ou la/les valeur(s) de $\mathrm{V}$;

- entrer dans un énoncé du type $\mathrm{V}$ est une valeur.

On peut aussi substituer à $\mathrm{V}$ son équivalent en termes de valorisation : le respect dû à $\mathrm{x}$, la supériorité de $\mathrm{x}$ sur $\mathrm{y}$. 


\section{Convocation} travers un mot ou une locution affectée d'un trait positif, de façon relativement stable. Pourvus d'une force illocutoire, leur mobilisation induit un dynamisme qui prouve la capacité du discours à s'orienter à partir du mot, par exemple dans ce texte célèbre, où l'auteur mobilise la «vérité » et la «justice», mais aussi la «juste gloire» et l'« honneur»:

Monsieur le Président,

Me permettez-vous, dans ma gratitude pour le bienveillant accueil que vous m'avez fait un jour, d'avoir le souci de votre juste gloire et de vous dire que votre étoile, si heureuse jusqu'ici, est menacée de la plus honteuse, la plus ineffaçable des taches? (...) Puisqu'ils ont osé, j'oserai aussi, mais la vérité, je la dirai, car j'ai promis de la dire, si la justice, régulièrement saisie, ne le faisait pas, je ne voudrais pas être complice... Et c'est à vous, Monsieur le Président, que je la crierai, cette vérité, de toute la force de ma révolte d'honnête homme. Pour votre honneur, je suis convaincu que vous l'ignorez...

(Émile Zola, « Lettre ouverte au Président de la République », L’Aurore, 1898)

\section{Promotion}

Les valeurs peuvent aussi être préconisées, voire prescrites. Il peut alors être utile de convaincre de la « valeur de la valeur » en justifiant sa prévalence ou en développant à son sujet une argumentation, au besoin de manière paradoxale :

Dire la vérité, toute la vérité, rien que la vérité, dire bêtement la vérité bête, ennuyeusement la vérité ennuyeuse, tristement la vérité triste : voilà ce que nous nous sommes proposé depuis plus de vingt mois, et non pas seulement pour les questions de doctrine et de méthode, mais aussi, mais surtout pour l'action. (Charles Péguy, Lettre du Provincial, 21 décembre 1899)

\section{Évocation}

Bien entendu les valeurs et les préférences ne sont pas toujours nommées, désignées, comme telles, mais apparaissent de manière indirecte à travers les traits saillants et les contrastes du texte, par exemple à travers les tensions, dans la description du jardin de Julie dans La Nouvelle Héloïse de Rousseau, entre la nature et l'artifice, la liberté et la contrainte, le désordre et l'ordre.

\section{Mention}

Enfin, dans le cas où elles se bornent à décrire un parti pris, une tendance constatée dans un groupe ou une société, les valeurs n'entrent pas directement dans l'argumentation de l'auteur. Elles informent sur une préférence individuelle ou collective remarquée par l'observateur, dans des termes qui peuvent être ceux d'un historien ou d'un sociologue. 
Évocation

(valeurs implicites)

Invocation

(valeurs brandies)
Préconisation

(valeurs promues

ou argumentées)
Convocation

(valeurs mobilisées)
Mention

(valeurs mentionnées ou décrites)

Argumentateur

Destinataire

Fig. 2 Fonctions pragmatiques des mots-valeurs

\section{Contenu sémantique des mots-valeurs} etc.), une dimension de l'expérience privilégiant certains types d'acteurs (la famille, la jeunesse), certaines relations au temps ou à l'espace (la tradition, le progrès, le changement, le terroir, le nomadisme).

\section{Valeurs et valorisation}

Mais les valeurs portées par les maîtres-mots ne doivent pas être détachées des valorisations ou dévalorisations auxquelles elles renvoient et qui interviennent sous des formes extrêmement variées dans le discours (adjectifs, verbes, adverbes) de manière directe ou indirecte : la "liberté » pourra par exemple être indirectement évoquée à travers la construction d'un réseau lexical accumulant des marqueurs dépréciatifs de soumission, de servitude, de domination. La mise en évidence des oppositions lexicales qui structurent les textes est aussi importante que les marques 
directes de l'éloge ou du blâme, puisque c'est essentiellement par différence et par contraste que le sens se construit.

On peut être légitimement déconcerté et découragé par la multiplicité quasi infinie de ces différences et renoncer à construire un cadre de référence permettant de se repérer dans ce chaos. Pourtant, un grand nombre d'oppositions récurrentes sont susceptibles d'être classées selon les grands paramètres de la mise en discours et favoriser ainsi notre attention aux singularités des textes. En effet quand on parle ou qu'on écrit, on mobilise toujours des sujets ou des forces (qui peuvent être humains ou non-humains), des verbes, dont certains peuvent être retenus pour leur caractère de grande généralité (être, avoir, faire, dire), des auxiliaires modaux (pouvoir, vouloir, devoir, etc.), ainsi que des termes qui alimentent des jugements de qualité, de quantité, ou des évaluations concernant l'espace et le temps. Ces oppositions courantes servent de support à la valorisation ou à la dévalorisation, que les contenus sémiques anti-orientés soient présents ou présupposés.

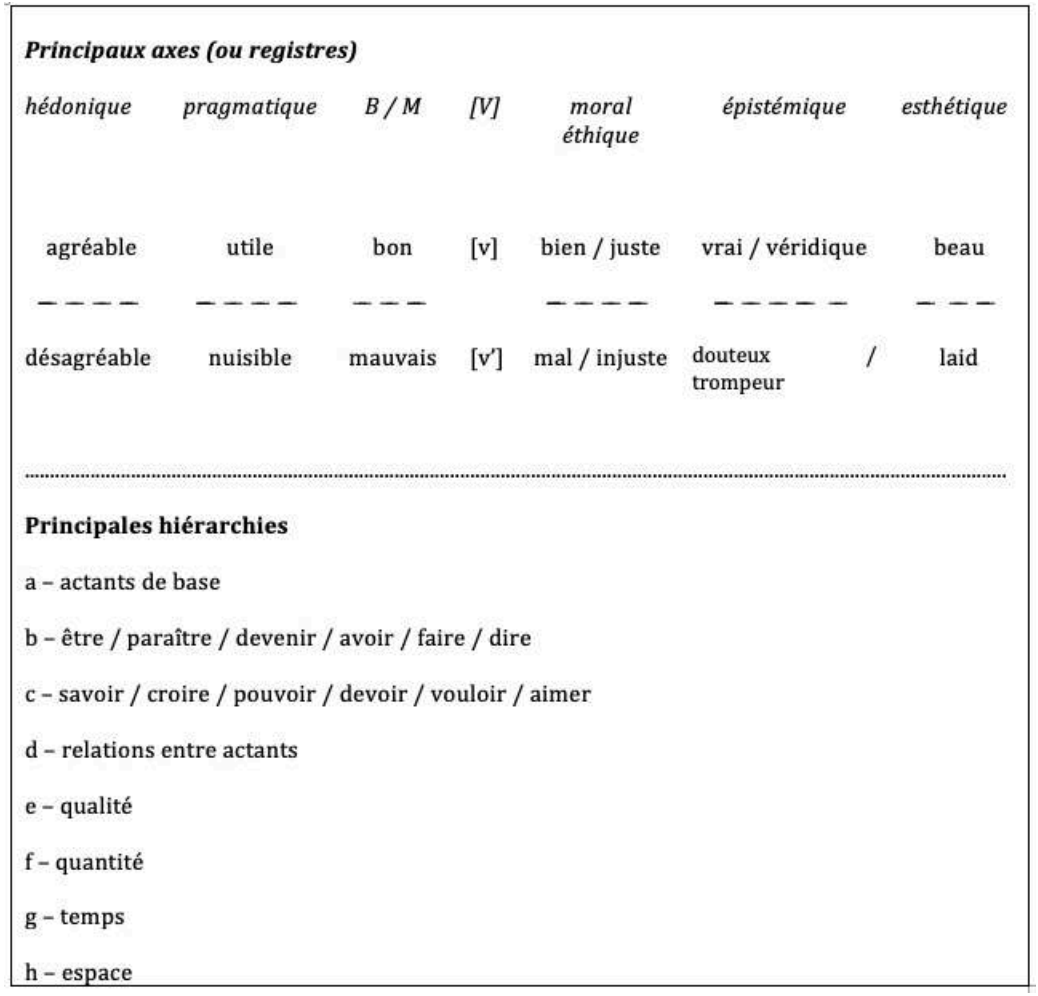

Figure 3 : Supports principaux de la valorisation et de la dévalorisation

Nous nous arrêterons seulement sur deux rubriques de ce tableau: les actants (les forces agissantes) et les relations actantielles.

On peut distinguer quatre catégories principales d'actants représentées sur la figure 4. Le principe de la distribution qu'il propose est simple et consiste à mettre en tension ses constituants : tout individu humain (représenté ci-dessous par l'esperluette placée au centre du schéma) se trouve sans cesse placé devant des priorités et des préférences. Il doit nécessairement prendre en compte trois dimensions de l'existence : d'une part son interaction avec des êtres non-humains, comme les choses de la nature, les êtres non-humains vivants ou non, les objets qu'il produit (point 3); d'autre part son interaction avec les êtres humains, qu'il s'agisse du plus proche ou du plus lointain 
(point 2) ; enfin les exigences de sa propre subjectivité (point 1). Quant au quatrième pôle, il indique l'aspiration humaine au sens, à la perfection ou du moins à un espoir d'amélioration. Ce peut être le lieu d'une croyance religieuse en un dieu (ou d'une aspiration spirituelle, mystique), ou plus modestement d'un effort humain vers un accomplissement personnel ou collectif. L'accent mis sur tel ou tel pôle, dans un contexte particulier et en fonction des investissements précis dont ils sont l'objet, implique des valorisations qui s'expriment en termes de choix existentiels.

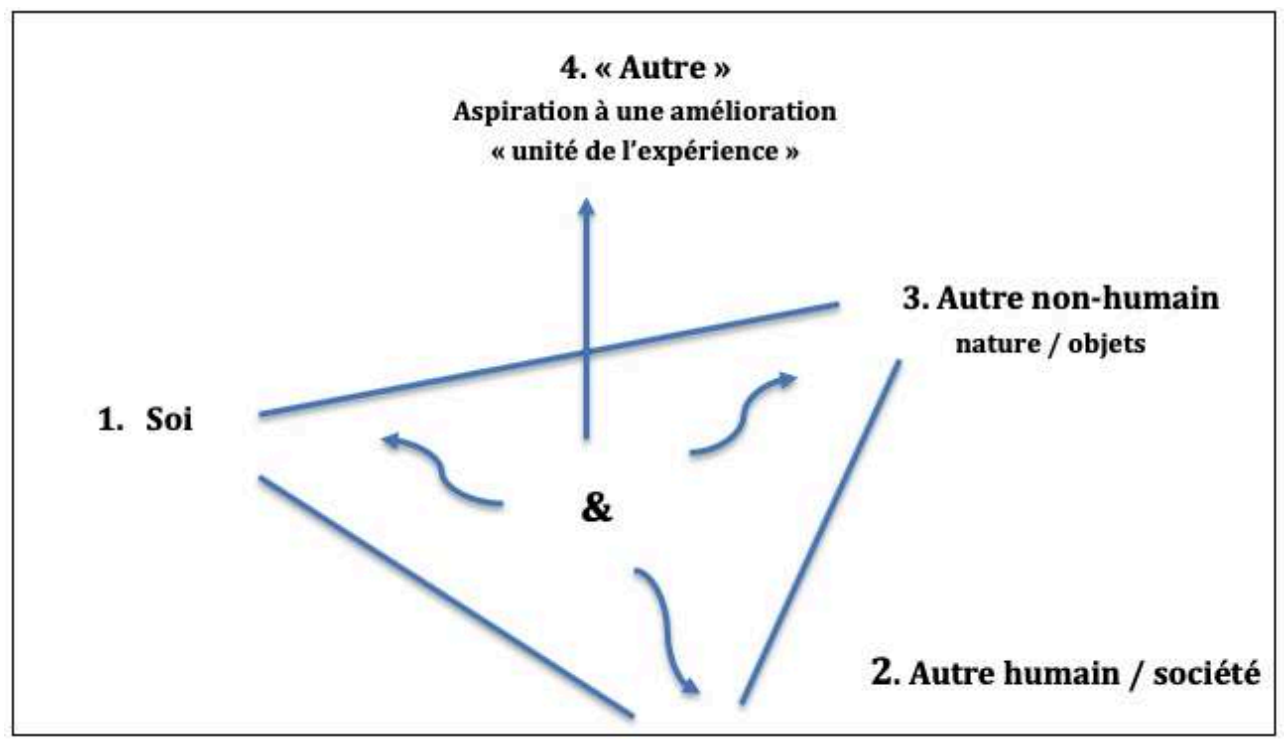

Figure 4 : Les actants de base

Le sujet (figuré par une esperluette, «\&») se trouve sollicité (tiraillé, déchiré, écartelé) par ces valorisations concurrentes et les dilemmes qu'elles suscitent.

Deuxième paramètre: les relations actantielles dont l'inventaire est virtuellement infini. Elles peuvent néanmoins donner lieu à une exploration méthodique dont nous indiquons une liste minimale :

\begin{tabular}{|l|l|}
\hline 1. & identité / similitude / ressemblance / différence / altérité \\
\hline 2. & union / séparation / mélange \\
\hline 3. & contrainte / dépendance / liberté \\
\hline 4. & domination / soumission / rébellion \\
\hline 5. & hiérarchie / complémentarité / égalité / réciprocité \\
\hline 6. & obligation / contrôle / laisser faire / autonomie \\
\hline 7. & accord / désaccord / négociation \\
\hline 8. & conflit / coexistence / coopération \\
\hline 9. & compétition / émulation / entraide \\
\hline
\end{tabular}




\begin{tabular}{|l|l|}
\hline 10. & destruction/ menace / protection \\
\hline & etc. \\
\hline
\end{tabular}
ici s'appuie principalement sur les propriétés des discours argumentatifs. Mais il va de soi que l'étude des récits ou des pièces de théâtre, menée, entre autres, avec ces rudiments d'analyse actantielle, rejoint notre propos. En effet, l'objectif poursuivi par le narrateur ou le dramaturge, est le plus souvent de rendre compte de la manière dont des personnages, au fil des tensions et des conflits qu'ils traversent, sont à la poursuite de formes de vie qui sont autant de manières d'affirmer ou de rejeter, de façon plus ou moins explicite, plus ou moins consciente, des partis pris de nature axiologique ${ }^{24}$. On voit tout l'intérêt qu'il y aura dans cette perspective à relier l'attention aux valeurs à l'expression des émotions, des passions et des sentiments car ils y trouvent généralement leur source et leur justification. 


\section{NOTES}

1. L'importance de l'œuvre de Chaïm Perelman (1913-1984) est désormais reconnue tant dans l'aire francophone qu'au niveau international. Voir notamment Marc Fumaroli, Histoire de la rhétorique dans l'Europe moderne, Paris, Presses universitaires de France, 1999; John Gage, The Promise of Reason: Studies in the New Rhetoric, Carbondale and Edwardsville, Southern Illinois University Press, 2011.

2. A. Compagnon, « Martyre et résurrection de Sainte Rhétorique », dans B. Cassin (dir.), Le Plaisir de parler, Paris, Minuit, 1986, p. 157-172.

3. C. Perelman, L. Olbrechts-Tyteca, Traité de l'argumentation. La nouvelle rhétorique [1958], Bruxelles, Presse de l'université de Bruxelles, 1988.

4. Il n'était pas possible de présenter ici des propositions didactiques précises. On peut se reporter à J.-C. Guerrini et E. Majcherzack, L'Argumentation au pluriel. Polyphonie, valeurs, points de vue, Classes des lycées et premier cycle universitaire, Lyon, Presses universitaires de Lyon, 1999, p. 39-122.

5. J.-Y. Château, Eutyphron, Philosophie et religion, 2006, p. 80.

6. N. Loraux, « Éloge de l'anachronisme en histoire ", Le Genre humain, n 27, 1993, p. 23-39.

7. Le mot axiologie apparaît en France en 1902 sous la plume du philosophe Paul Lapie qui prétendait élaborer une « Science de la Valeur ».

8. C. Kerbrat-Orecchioni, « Déambulation en territoire aléthique », in Stratégies discursives, Lyon, Presses universitaires de Lyon, 1978 ; L'Énonciation. De la subjectivité dans le langage, Paris, A. Colin, 1980.

9. L. Gosselin, Les Modalités en français. La validation des représentations, Amsterdam, New York, Rodopi, 2010.

10. Ibid., p. 52.

11. Ibid., p. 53.

12. Ibid., p. 28.

13. Ibid., p. 33, italiques de l'auteur.

14. P. Charaudeau, Grammaire du sens et de l'expression, Paris, Hachette éducation, 1992.

15. Ibid., p. 814, italiques de l'auteur.

16. C'est pour cette raison que $\mathrm{N}$. Heinich préfère le terme de registre. Mais il se trouve que ce dernier est affecté dans les études littéraires à un autre usage qui nous paraît utile (registre comique, tragique, etc.). Dès l'instant que le contexte est suffisamment clair, rien n'empêche d'user, selon les cas, d'axe ou de registre.

17. Nous adoptons ici cette graphie, à la suite de Kerbrat-Orecchioni (ouvr. cité, 1980, p. 75).

18. Les Modalités en français, ouvr. cité, p. 338.

19. J. Swift, Modest proposal For Preventing the Children of Poor People From Being a Burden to Their Parents or Country, and For Making them Beneficial to the Public [1729], traduit en français par Modeste proposition pour empêcher les enfants des pauvres d'être à charge à leurs parents ou à leur pays et pour les rendre utiles au public, Paris, Mille et une nuits, 2005.

20. Les modalités appréciatives de L. Gosselin chevauchent trois axes, ce que mentionnent les crochets.

21. Bien entendu, il n'y a pas de réponse "scientifique " à ces questions. On est contraint à une discussion sur ce qui est valeur ou non valeur dans telle situation, pour les acteurs concernés.

22. On peut trouver une palette plus développée du tableau et de ces paramètres dans Guerrini et Majcherczak, 1999, ouvr. cit. p. 81. 
23. Un inventaire de 231 mots-valeurs distribués selon ce principe est présenté dans J.C. Guerrini, Les valeurs dans l'argumentation. Structures axiologiques et dimension axiologique des disputes. Thèse de doctorat soutenue à l'Université Lyon 2, 2015, p. 464.

24. Voir M. Macé, Styles. Critique de nos formes de vie, Paris, Gallimard, 2016.

\section{RÉSUMÉS}

Pour être en mesure de transmettre et de questionner les valeurs, il est bon de savoir comment on les identifie, comment elles entretiennent des relations de différence et de proximité, bref, comment on peut avoir une idée d'ensemble de la dimension axiologique des discours. Le propos de cet article est de montrer que la question des valeurs peut être abordée à partir de ce qui constitue le cœur de la discipline du français : l'enseignement de la langue et l'étude des textes. Mots-valeurs, valorisation (et dévalorisation) au travers des axes sémantiques et des supports principaux de l'évaluation dans l'activité discursive donnent lieu à une enquête méthodique qui fait apparaître des constantes et favorise du même coup l'accès à la singularité des textes.

To be able to transmit and question the values, it is good to know how they are identified, how they maintain relations of difference and proximity, in short, how one can have an overall idea of the axiological dimension of the speeches. This paper aims to show that the question of values can be approached from what constitutes the heart of the discipline of French: the teaching of language and the study of texts. Values, valorization (and depreciation) through the semantic axes and the main supports of the evaluation in the discursive activity give place to a methodical investigation which brings up constants and at the same time favors the access to the singularity of the texts.

\section{AUTEUR}

\section{JEAN-CLAUDE GUERRINI}

Jean-Claude Guerrini, agrégé de Lettres modernes, est retraité de l'Éducation nationale. Il a publié en 1999, en collaboration avec Edmond Majcherczak, L'Argumentation au pluriel. Polyphonie, valeurs, points de vue (Presses universitaires de Lyon). Il a soutenu en 2015 une thèse dirigée par Christian Plantin intitulée Les Valeurs dans l'argumentation. Structures axiologiques et dimension axiologique des disputes. Il est actuellement chercheur associé au laboratoire ICAR (ENS-CNRSUniversité de Lyon). 